\title{
Quasicrystalline compactification
}

\section{Citation}

Harvey, J., G. Moore, and C. Vafa. 1988. "Quasicrystalline Compactification." Nuclear Physics B 304 (January): 269-90. https://doi.org/10.1016/0550-3213(88)90627-x.

\section{Permanent link}

http://nrs.harvard.edu/urn-3:HUL.InstRepos:41385045

\section{Terms of Use}

This article was downloaded from Harvard University's DASH repository, and is made available under the terms and conditions applicable to Other Posted Material, as set forth at http:// nrs.harvard.edu/urn-3:HUL.InstRepos:dash.current.terms-of-use\#LAA

\section{Share Your Story}

The Harvard community has made this article openly available.

Please share how this access benefits you. Submit a story.

Accessibility 


\title{
QUASICRYSTALLINE COMPACTIFICATION
}

\author{
J. HARVEY \\ Princeton University, Princeton, NJ 08540, USA \\ G. MOORE \\ Institute for Advanced Study, Princeton, NJ 08540, USA \\ C. VAFA \\ Lyman Laboratory of Physics, Harvard University, Cambridge, MA 02138, USA \\ Received 14 October 1987

\begin{abstract}
A class of asymmetric orbifolds is constructed using ideas from the theory of quasicrystals. These orbifolds provide examples of solutions to string theory which are isolated in the sense that they do not belong to a continuous moduli space of solutions and moreover cannot be approximated by rational orbifolds. One of the notable features of the construction is that many aspects of the models are easily handled with the theory of cyclotomic fields.
\end{abstract}

\section{Introduction}

The space of two-dimensional conformal field theories, $\mathscr{C}$, is of interest for its own sake and for its usefulness in string theory. Two issues regarding this space have attracted a certain amount of attention: its topology and its conjectured arithmetic nature. In this paper we examine a class of asymmetric orbifolds whose properties shed some light on these issues.

It has been conjectured [1] that $\mathscr{C}$ is in some sense an arithmetic space. Moreover, it has been proposed that the arithmetic nature of this space might even provide a useful approximation scheme in string theory. Let us briefly discuss this. Conformal theories give rise to flat projective vector bundles on the moduli space of Riemann surfaces. For example, a theory of free Majorana fermions gives rise to a $2^{2 g}$-dimensional bundle over the moduli space of genus $g$ Riemann surfaces. Flat projective vector bundles on moduli space are in one-to-one correspondence with projective representations of the mapping class group. Conformal theories which give rise to finite-dimensional bundles over the moduli space are useful because they correspond to finite-dimensional representations of the mapping class group. One may 
hope to get an understanding of the full space of conformal theories by first understanding the finite-dimensional case and then somehow completing this space to obtain the full space [1].

Conformal theories which give rise to finite-dimensional bundles over the moduli space may be called "rational conformal field theories" (RCFTs). Let us describe why the appelation "rational" is appropriate $[1,2]$. Consider the partition function of the bosonic string compactified on a torus defined by a $d$-dimensional lattice $\Lambda^{\star}$

$$
Z=\frac{1}{|\eta|^{2 d}} \sum q^{p_{\mathrm{L}}^{2} / 2} \bar{q}^{p_{\mathrm{R}}^{2} / 2}
$$

with

$$
\begin{array}{ll}
p_{\mathrm{L}}=p / 2+w & p \in \Lambda^{*}, \\
p_{\mathrm{R}}=p / 2-w & w \in \Lambda .
\end{array}
$$

The left- and right-moving momenta belong to the set of points $\Gamma=\frac{1}{2} \Lambda^{*}+\Lambda \subseteq \mathbf{R}^{d}$. This set of points defines a lattice if and only if the quadratic form of the lattice is rational $^{\star \star}$. If $\Gamma$ is a lattice, then its dual $\Gamma^{*}=2 \Lambda \cap \Lambda^{*}$ is a sublattice and we may consider $p_{\mathrm{L}}, p_{\mathrm{R}}$ as vectors in $\Gamma^{*}$ shifted by elements of the coset space $\Gamma / \Gamma^{*}$. Thus, we may rewrite for the partition function on the torus with modular parameter $\tau$

$$
Z=\frac{1}{|\eta|^{2 d}} \sum_{\alpha \in \Gamma / \Gamma^{*}, \beta \in \Gamma / \Gamma^{*}} \vartheta_{\Gamma^{*}}\left[\begin{array}{l}
\alpha \\
0
\end{array}\right] \mathscr{M}_{\alpha \beta} \bar{\vartheta}_{\Gamma^{*}}\left[\begin{array}{c}
\beta \\
0
\end{array}\right]
$$

where

$$
\mathscr{M}_{\alpha \beta}= \begin{cases}1 & \alpha+\beta \in \Lambda^{*}, \alpha-\beta \in 2 \Lambda \\ 0 & \text { otherwise }\end{cases}
$$

and $\vartheta_{\Gamma^{*}}$ is the theta function for the lattice $\Gamma^{*}$. This means that the partition function is of the form

$$
Z(\tau, \bar{\tau})=\sum_{i=1}^{N} f_{i}(\tau) \bar{g}_{i}(\tau)
$$

where $f_{i}, g_{i}$ are holomorphic and $N<\infty$. This gives rise to a finite-dimensional bundle over the moduli space of genus one curves. Moreover it can be shown, using the results in [3], that for arbitrary genus the partition function for such a rational

* For simplicity we ignore the possibility of turning on background antisymmetric tensor fields. The result generalizes to this case.

$\star \star$ The reader might enjoy proving this not completely trivial statement. The notion of the quadratic form being rational is sensible because the lattice breaks rotational symmetry to a subgroup of $\mathbf{G L}(d, \mathbf{Z})$. Similarity transformations by this group preserve the property of rationality. 
toroidal compactification is a finite sum of holomorphic times antiholomorphic pieces, giving rise to a finite-dimensional bundle over the moduli space. The moral of the story is that the rationality of the background data leads to finite-dimensional bundles.

Tori which correspond to irrational background data give rise to infinite-dimensional bundles over the moduli space. But we know that this infinity is in some sense trivial, because by a tiny change of the background data from irrational to rational, the infinite-dimensional bundle collapses to a finite-dimensional one. Thus, with any reasonable definition, the "completion" of the space of conformal theories for rational tori will include the irrational tori and this implies that for conformal theories with toroidal target space, RCFTs which give rise to finite-dimensional bundles are "dense" in the space of all conformal theories.

Our main goal in this paper is to show that there are irrational conformal theories which are not "close" to any rational conformal theories. We will not quite be able to show this, partly because the meaning of "close" is not completely clear, and partly because the structure of the space of conformal theories is not well understood. Although the understanding of the space of conformal theories has not yet been developed to a point where one has a clear understanding of the topology of $\mathscr{C}$, one can still discuss what it means for two conformal theories to be "close". One way to deform a conformal theory is to give an expectation value to an operator of dimension $(1,1)$. Note that these operators have the right dimension to be integrated over the world surface, so that the procedure is coordinate independent. However, giving an expectation to such an operator does not in general preserve the conformal structure, since quantum corrections due to the perturbation can spoil conformal invariance. Thus, the number of conformal deformations which may be accomplished in this way is less than or equal to the number of dimension $(1,1)$ operators in the theory (these operators correspond to massless particles in spacetime; the self-interactions of the massless scalars can destroy conformal invariance). Moreover, it is believed that all the deformations of conformal theories (apart from an overall choice of coupling constant) may be obtained in this way so that the dimension of the tangent space to $\mathscr{C}$ at some theory is less than or equal to the number of massless scalars. Thus, if some conformal theory $X \in \mathscr{C}$ contains no dimension $(1,1)$ operators, one might expect that in a reasonable topology for $\mathscr{C}, X$ would be an isolated point.

There are two senses in which two conformal theories may be said to be "close". A strong sense of closeness may be defined by requiring that both theories lie on a continuous line of conformal theories obtained by giving some expectation value to a dimension $(1,1)$ operator. If the expectation value is small then the theories would be close, and continuously connected. For example, two toroidal compactifications with slightly different toroidal radii are close in the strong sense. A weak sense of closeness can be defined by requiring that there is a one-one correspondence of primary fields with approximately equal weights such that the two conformal 
theories have operator product expa intuitively clear that a theory which is close in the strong sense is close in the weak sense. In both cases we may define two conformal theories to be within distance $\varepsilon$ if, under the correspondence $\phi_{x}^{i} \leftrightarrow \phi_{y}^{i}$ for the primary fields in theories $x, y$ we have for conformal weights and operator product-expansion coefficients

$$
\begin{array}{r}
\left|h_{x}^{i}-h_{y}^{i}\right|<\varepsilon, \\
\left|C_{j k}^{i}(x)-C_{j k}^{i}(y)\right|<\varepsilon .
\end{array}
$$

Many variations on the definition (1.5) can be contemplated (in the above definition one should require a uniform bound only for a finite number of fields at a time).

By examining some special examples of asymmetric orbifolds [4] which are based on irrational tori we will prove that there are some irrational conformal theories which are not close to any conformal theory in the strong sense. Moreover, we will show that in the weak sense of "close" there are no close rational orbifolds. This does not imply that there are not any close conformal theories in the weak sense, since orbifolds are very special conformal theories. Nevertheless, one would naively expect that rational orbifolds are the best candidates for approximating irrational orbifolds. Since this is not the case there is some doubt as to whether there are any RCFTs weakly close to the ones we describe. Thus, if RCFTs can be used to approximate arbitrary conformal field theories they must do so in a more subtle way.

Some alternative definitions of what rational conformal theories could be are described in the appendix.

\section{Asymmetric orbifolds}

We begin by reminding the reader of some elementary facts about asymmetric orbifolds. The reader should consult [4] for a more complete exposition. In a toroidal compactification $[5,6]$ left- and right-moving fields form coordinates on $R^{p, q} / \Gamma^{p, q}$ where $\Gamma^{p, q}$ is an even self-dual lorentzian lattice of signature $(p, q)$, and hence $p-q=0(8)$ (we shall use the notation of a number inside a parenthesis to mean "modulo that number"). For the bosonic string we take $p=q=d$.

The Hilbert space of the toroidal compactification is obtained by acting on momentum eigenstates $\left|p_{\mathrm{L}}, p_{\mathrm{R}}\right\rangle$ with oscillators. To form an orbifold we twist by a symmetry group $\mathrm{G}$ of the theory. Elements of $\mathrm{G}$ are pairs $(a, \theta)$ where $a$ is a shift vector and $\theta$ is an automorphism of $\Gamma$ which commutes with the hamiltonian $H=\frac{1}{2} p_{\mathrm{L}}^{2}+\frac{1}{2} p_{\mathrm{R}}^{2}+N_{\mathrm{L}}+N_{\mathrm{R}}$ so $\theta \in \mathrm{O}(d) \times \mathrm{O}(d) \subset \mathrm{O}(d, d)$. The Hilbert space representation of $\theta$ acts by

$$
\theta\left|p_{\mathrm{L}}, p_{\mathrm{R}}\right\rangle=\mathrm{e}^{2 \pi i p \cdot a}\left|\theta_{\mathrm{L}} p_{\mathrm{L}}, \theta_{\mathrm{R}} p_{\mathrm{R}}\right\rangle
$$


In a symmetric orbifold, $p_{\mathrm{L}}, p_{\mathrm{R}}$ belong to isomorphic spaces and $\theta_{\mathrm{L}}=\theta_{\mathrm{R}}$. Let us discuss the condition for modular invariance for asymmetric orbifolds. To describe this let $I$ denote the sublattice of $\Gamma$ invariant under $\theta$. The basis for $I$ has as a real span some copy of $\mathbf{R}^{k}$. Let $I^{*}$ be the dual of $I$ in this ambient space. Then modular invariance, together with the requirement that there be an integral number of ground states in the twisted sectors, implies that

$$
\sqrt{\frac{\operatorname{det}^{\prime}(1-\theta)}{\left|I^{*} / I\right|}} \in \mathbf{Z}, \quad \theta \in \mathrm{G},
$$

(where $\operatorname{det}^{\prime}$ means deleting the zero eigenvalues in taking the determinant). In fact, as shown in [4], (2.2) is always satisfied for $\Gamma_{d, d}$ even, self-dual. In our constructions, however, we do not start from a given self-dual lattice, but rather we start from a rotation matrix $\theta$ and try to construct a self-dual, even Lorentzian lattice which admits $\theta$ as an automorphism. Thus, given a putative $\theta$ and $I,(2.2)$ is a necessary condition for $\theta$ to be an automorphism of an even self-dual $\Gamma_{d, d}$ with invariant lattice $I$. This condition must be satisfied for every element of the orbifold group.

The main condition for modular invariance is level matching. If $\theta$ has order $n$ then the difference of energies for left- and right-movers must satisfy

$$
n\left(E_{\mathrm{L}}-E_{\mathrm{R}}\right)=0(1) \text {. }
$$

We denote the complex eigenvalues of $\theta_{\mathrm{L}}, \theta_{\mathrm{R}}$ by

$$
\begin{aligned}
& \theta_{\mathrm{L}}=\operatorname{diag}\left\{\mathrm{e}^{2 \pi i r_{1}} \ldots \mathrm{e}^{2 \pi i r_{k}} ; \text { c.c. }\right\}, \\
& \theta_{\mathbf{R}}=\operatorname{diag}\left\{\mathrm{e}^{2 \pi i l_{1}} \ldots \mathrm{e}^{2 \pi i l_{k}} ; \text { c.c. }\right\},
\end{aligned}
$$

where $^{\star} k=\frac{1}{2} d$. The condition (2.3) implies (for bosonic strings) that

$$
\sum r_{i}^{2}=\sum l_{i}^{2} \begin{cases}\bmod n & n \text { odd } \\ \bmod 2 n & n \text { even }\end{cases}
$$

and if the order of the twist is even then we must also have

$$
v \theta^{n / 2} v=0(2)
$$

for every vector $v$ in the lattice (for simplicity we have taken $a=0$ ). It has been shown in [7] that, in the case of (abelian) symmetric orbifolds, level matching is sufficient to give modular invariance to all loops (and that for the non-abelian case there are some additional constraints). For asymmetric orbifolds, one can show that level matching is sufficient for modular invariance at one loop [8]. One expects that

\footnotetext{
* We will always consider compactifications of even numbers of dimensions for simplicity.
} 
for abelian asymmetric orbifolds, this should be sufficient to all loops, but this has not been shown yet. For fermionic string theories the level matching conditions are slightly different (for heterotic strings the right-hand side of (2.5) is absent, and there is an additional mod 2 condition on the sum of the eigenvalues on the right, and for type II superstrings there are only mod 2 conditions (2.6) and the vanishing of the sum of the eigenvalues mod 2 on each side (remember that we are taking $a=0)$ ).

We would like to construct some asymmetric orbifolds which have few massless particles and which are, in some sense, far from rational orbifolds. Both criteria can be met if we borrow an idea from the theory of quasicrystals. There, one uses the fact that a $p+q$ dimensional lattice $\Lambda^{p, q}$ can have an automorphism whose order is not allowed in $p$ or $q$ dimensions. Subsets of the projection of $\Lambda^{p, q}$ on $p$-dimensional space form the vertices of an aperiodic tiling. The most common example in solid state physics is $\mathbf{Z} / 5 \mathbf{Z}$ symmetry which is allowed in four, but not in two or three dimensions. We will use analogs of this construction to produce interesting asymmetric orbifolds. We call these quasicrystalline orbifolds. In this way, the projection of the momenta to left- and right-moving components in the untwisted sector will give irrational vectors, and the acrystallographic symmetry on left and right exists precisely because of the existence of these irrational vectors. We will see later, through some examples, how in some cases these constructions can get rid of the massless moduli as well.

Consider the case of a cyclic orbifold group $G=\mathbf{Z} / m \mathbf{Z}$. We would like to produce a $\mathbf{Z} / m \mathbf{Z}$ twist which acts crystallographically on $\Gamma_{d, d}$, yet cannot act crystallographically in $d$ dimensions. Let the generator of $\mathrm{G}$ be the automorphism $\theta \in \mathrm{O}(d) \times \mathrm{O}(d)$. In a lattice basis, $\theta$ must have integral entries so there is a matrix $A \in \mathrm{GL}(2 d, \mathbf{R})$ such that $A \theta A^{-1} \in \mathrm{GL}(2 d, \mathbf{Z})$. If we diagonalize the rotation we can write

$$
\begin{gathered}
\theta_{\mathrm{L}}=\operatorname{diag}\left(\xi^{i_{1}} \ldots \xi^{i_{d}}\right), \\
\theta_{\mathrm{R}}=\operatorname{diag}\left(\xi^{i_{d+1}} \ldots \xi^{i_{2 d}}\right),
\end{gathered}
$$

where we group the phases so that $\theta_{\mathrm{L}}, \theta_{\mathrm{R}}$ are both similar to orthogonal matrices by the standard transformation $M \oplus \ldots \oplus M$ with

$$
M=\frac{1}{2}\left(\begin{array}{cc}
1 & i \\
1 & -i
\end{array}\right) .
$$

We will show that it is relatively easy to construct rotations of the kind we are seeking if we take the phases to be primitive $m$ th roots of unity. The constraints (2.2) and (2.5) and the construction of lattices are all relatively easy once the problem is put in the proper setting. That setting turns out to be the theory of cyclotomic fields, which we now briefly describe. 


\section{Some elementary number theory}

\subsection{CYCLOTOMIC FIELDS}

A primitive $m$ th root of unity is an $m$ th root of unity $\xi$ such that $m$ is the smallest natural number for which $\xi^{m}=1$. For example, we could take $\xi=\mathrm{e}^{2 \pi i / m}$. The $m$ th cyclotomic field is simply the extension of $\mathbf{Q}$ by $\xi: \mathbf{Q}[\xi]$. Concretely, elements of $\mathbf{Q}[\xi]$ are linear combinations

$$
\alpha=\sum_{i=0}^{m-1} a_{i} \xi^{i}, \quad a_{i} \in \mathbf{Q}
$$

The basic tool for working with cyclotomic fields is the cyclotomic polynomial

$$
\Phi_{m}(x)=\prod_{(a, m)=1}\left(x-\xi^{a}\right) .
$$

The basic facts about $\Phi_{m}$, which one can find proved in elementary texts on number theory $[9,10]$ are that

(1) $\Phi_{m}(x) \in \mathbf{Z}[x]$. That is, the coefficients of $\Phi_{m}(x)$ are in $\mathbf{Z}$.

(2) $\Phi_{m}(x)$ is irreducible in $\mathbf{Z}[x]$.

(3) $x^{m}-1=\prod_{d \mid m} \Phi_{d}(x)$.

Property three allows one to calculate the $\Phi_{m}$ recursively. Some of the low order polynomials are

$$
\begin{aligned}
& \Phi_{1}(x)=x-1, \\
& \Phi_{2}(x)=x+1, \\
& \Phi_{4}(x)=x^{2}+1, \\
& \Phi_{6}(x)=x^{2}-x+1, \\
& \Phi_{8}(x)=x^{4}+1, \\
& \Phi_{9}(x)=x^{6}+x^{3}+1, \\
& \Phi_{10}(x)=x^{4}-x^{3}+x^{2}-x+1, \\
& \Phi_{12}(x)=x^{4}-x^{2}+1, \\
& \Phi_{15}(x)=x^{8}-x^{7}+x^{5}-x^{4}+x^{3}-x+1, \\
& \Phi_{18}(x)=x^{6}-x^{3}+1, \\
& \Phi_{24}(x)=x^{8}-x^{4}+1 .
\end{aligned}
$$


Note that the degree of $\Phi_{m}(x)$ is just the number of integers $0<a<m$ such that $(a, m)=1$ and $(a, m)$ is the greatest common divisor. This number is denoted $\phi(m)$ and $\phi$ is called the Euler totient function. $\phi$ is a very handy function for working with quasicrystalline orbifolds and the reader might enjoy proving the following very useful properties:

(i) If $(m, n)=1, \phi(m n)=\phi(m) \phi(n)$.

(ii) $\phi\left(p^{r}\right)=p^{r}-p^{r-1}$ for a prime $p$.

(iii) Therefore $\phi(m)$ is even, except for $m=2$ when $\phi=1$.

Another useful result is the following

Theorem.

$$
\Phi_{m}(1)= \begin{cases}p & \text { if } m=p^{r}, p \text { prime } \\ 1 & \text { otherwise }\end{cases}
$$

Proof. The theorem is easily verified for $m=p$, a prime, since $\Phi_{p}(x)=x^{p-1}$ $+\ldots+1$. In particular, it is true for $m=2,3$. We now decompose $m$ into prime factors $m=p_{1}^{e_{1}} \ldots p_{n}^{e_{n}}$ and proceed by induction on $N=e_{1}+\ldots+e_{n}$. Note that

$$
\Phi_{m}(1)=\frac{m}{\prod_{n \mid m, 1<n<m} \Phi_{n}(1)} .
$$

Therefore, if $m^{\prime}=p_{1}^{e_{1}} \ldots p_{n}^{e_{n}} p_{n+1}$ then

$$
\Phi_{m^{\prime}}(1)=\frac{p_{1}^{e_{1}} \ldots p_{n}^{e_{n}} p_{n+1}}{p_{1}^{e_{1}} \ldots p_{n}^{e_{n}} p_{n+1}}=1,
$$

and if $m^{\prime}=p_{1}^{e_{1}} \ldots p_{r}^{e_{r}+1} \ldots p_{n}^{e_{n}}$ we again compute $\Phi_{m^{\prime}}(1)=1$. If $m=p^{e}$ then we obtain directly $p$. QED.

\subsection{THE GROUP OF UNITS IN $(\mathrm{Z} / M \mathrm{Z})^{*}$}

Recall that if $a$ is relatively prime to $m$ then by the euclidean algorithm there exist integers $x, y$ such that $a x+m y=1$. Reducing $\bmod m$ we see that the residue classes of the $\phi(m)$ integers $a$ with $(a, m)=1$ form a group, known as the group of units $(\mathbf{Z} / m \mathbf{Z})^{*}$. Some standard results of number theory, which we use to prove level matching are

(1) If $m=p_{1}^{e_{1}} \ldots p_{n}^{e_{n}}$ for distinct primes then

$$
(\mathbf{Z} / m \mathbf{Z})^{*} \cong\left(\mathbf{Z} / p_{1}^{e_{1}} \mathbf{Z}\right)^{*} \times \ldots \times\left(\mathbf{Z} / p_{r}^{e_{r}} \mathbf{Z}\right)^{*}
$$

(2) If $p$ is an odd prime $\left(\mathbf{Z} / p^{e} \mathbf{Z}\right)^{*}$ is a cyclic group of order $\phi\left(p^{e}\right)=p^{e}-p^{e-1}$.

(3) If $p=2$ we have

$$
(\mathbf{Z} / 4 \mathbf{Z})^{*}=\langle \pm 1\rangle
$$


while for $e \geqslant 3$

$$
\left(\mathbf{Z} / 2^{e} \mathbf{Z}\right)^{*}=\langle \pm 1\rangle \times\langle a\rangle
$$

where $a$ has order $2^{e-2}$, and can be taken to be the residue class of 5 .

\section{Bosonic quasicrystals}

We now apply some of the number theory in the previous section to the construction of bosonic quasicrystals. In this section we will study the constraints of the existence of a crystallographic action and modular invariance. In sect. 5 we study an example.

We now consider the rotation (2.7) where the phases are primitive $m$ th roots of unity and $\phi(m)=2 d$. We consider these rotations because we can easily construct lattices which have these types of symmetries. However, we wish to emphasize that for having a quasicrystalline orbifold it is by no means necessary to start from such rotations and the idea that the left and right rotations are not separately crystallographic is more general. To decide on the crystallographic nature of the rotation (2.7) we consider the relation of $\operatorname{tr} \theta$ to the coefficients of the cyclotomic polynomial, $\Phi_{m}(x)$. From the definition (3.2) we see that the coefficients $a_{i}$ in

$$
\Phi_{m}(x)=x^{\phi(m)}+a_{1} x^{\phi(m)-1}+\ldots+a_{\phi(m)}
$$

are symmetric polynomials in the $\xi$ :

$$
\begin{aligned}
\operatorname{tr} \theta & =a_{1}, \\
\operatorname{tr} \theta^{2} & =a_{1}^{2}-2 a_{2} \ldots
\end{aligned}
$$

Since the $a_{i}$ are rational integers ${ }^{\star} \operatorname{tr} \theta^{k} \in \mathbf{Z}$ for all powers and $\theta$ can act crystallographically. Since $\Phi_{m}(x)$ is irreducible over $\mathbf{Z}[x]$, not all powers of $\operatorname{tr} \theta_{\mathrm{L}}^{k}$ can lie in $\mathbf{Z}$ so $\theta_{\mathrm{L}}$ cannot act crystallographically.

We now consider the restrictions (2.2), (2.5) and (2.6). Consider first the condition (2.2). Since (2.7) leaves no invariant lattice $\operatorname{det}(1-\theta)$ must be the square of an integer. Since $\operatorname{det}(1-\theta)=\Phi_{m}(1)$ we can use (3.4) to see that $m$ cannot be a power of a prime. This eliminates the possibilities $m=1,2,3,4,5,6,7,8,9,11,13$, $16,17,19, \ldots$. Furthermore, if $m=2 p^{r}$ for $p$ prime and $r \in \mathbf{Z}_{+}$then $\phi(m)=$ $\phi(2) \phi\left(p^{r}\right)=\phi\left(p^{r}\right)=\phi(m / 2)$. Thus, if $m=2 p^{r}$ then $\theta^{2}$ has no invariant lattice and $\operatorname{det}\left(1-\theta^{2}\right)=p$ violates (2.2). The first few $m$ allowed by (2.2) are therefore $m=12,15,20,21,24, \ldots$.

We now show that we do not obtain new conditions on $m$ by considering (2.2) for higher powers of $\theta$. If $\theta$ is built from $\phi(m)$ primitive $m$ th roots of unity then $\theta^{k}$ will contain primitive $(m /(k, m))$ th roots of unity raised to the $k /(k, m)$ th power. Since

"The adjective "rational" distinguishes ordinary integers from the ring of integers in an algebraic number field. 
$(m /(k, m))$ and $(k /(k, m))$ are relatively prime we see that $\theta^{k}$ contains $\phi(m) / \phi[(m /(k, m))]$ sets of primitive $(m /(k, m))$ th roots of unity. If the prime decomposition of $(m /(k, m))$ contains more than one distinct prime then $\operatorname{det}\left(1-\theta^{k}\right)=1$ by (3.4). If $m=p_{1}^{e_{1}} \ldots p_{n}^{e_{n}}$ and $(m /(k, m))=p_{j}^{e_{j}-s_{j}}$ then

$$
\frac{\phi(m)}{\phi[(m /(k, m))]}=\prod_{i \neq j}\left(p_{i}^{e_{i}}-p_{i}^{e_{i}-1}\right) \cdot p_{j}^{s_{j}} .
$$

The above product is always even except possibly in the case that $m=2 p^{r}$, but this case has already been excluded. Since the ratio is even $\operatorname{det}\left(1-\theta^{k}\right)$ is a square.

We next consider the condition of level matching (2.5). We will show that if $m$ is not of the form $p^{r}$ or $2 p^{r}$ then there always exists an ordering of $\xi^{i_{k}}$ in (2.7) so that level matching is satisfied. The details of the argument are a bit technical, and the reader who is willing to accept this statement should skip the next three paragraphs.

The integers $0<i_{1} \ldots<i_{\phi(m)}<m$ relatively prime to $m$ have residue classes $\bmod m$ which are invertible. These form the group of units $(\mathbf{Z} / m \mathbf{Z})^{*}$ in $\mathbf{Z} / m \mathbf{Z}$. We now consider separately the cases $m$ odd and even.

If $m$ is odd we are interested in the solutions of the equation

$$
x^{2}=r^{2} \quad \bmod m
$$

for $x$. Since $r$ is relatively prime to $m$ any solution will be also, and hence will be invertible. Thus, the problem reduces to finding solutions to (4.2) with $r=1$. Writing $m=p_{1}^{e_{1}} \ldots p_{n}^{e_{n}}$ for $e_{i}>0$ and odd primes $p_{i}$ we know from subsect. 3.2 that $(\mathbf{Z} / m \mathbf{Z})^{*}$ is a product of cyclic groups with generators $a_{i}, i=1, \ldots, n$, of order $\phi\left(p_{i}^{e_{i}}\right) \equiv \phi_{i}$. Thus, (4.2) has exactly $2^{n}$ solutions, namely, if we define $u_{i} \equiv a_{i}^{\phi_{i} / 2}$ then $x=u_{l}^{\eta_{1}} \ldots u_{n}^{\eta_{n}} r$ is a solution to (4.2) if $\eta_{i} \in\{0,1\}$. Moreover, $x$ is a solution iff $(m-x)$ is a solution and since $x$ is invertible, these solutions are distinct. Therefore, there are $2^{n-1}$ solutions to (4.2) with $x<[m / 2]$. For $n>1$ (the condition that $m$ is not a power of a prime) the number of such solutions is divisible by two. Thus, in the list $i_{k}^{2} \bmod m, k \leqslant \phi(m) / 2$ each entry appears an even number of times, so we can "distribute" the $i_{k}^{\prime}$ s on the left and right to satisfy level matching.

If $m$ is even we have $m=2{ }^{e} p_{1}^{e_{1}} \ldots p_{n}^{e_{n}}$ with $e \geqslant 2$ and we are interested in solutions to

$$
x^{2}=r^{2} \bmod 2 m
$$

for $r \in(\mathbf{Z} / m \mathbf{Z})^{*}$. Notice that if $x \in(\mathbf{Z} / m \mathbf{Z})$ it does not define a residue class in $(\mathbf{Z} / 2 m \mathbf{Z})$, rather $(\mathbf{Z} / 2 m \mathbf{Z})$ is a twofold cover of $\mathbf{Z} / m \mathbf{Z}$ and a residue has two lifts, $x, x+m$. If $m$ is even this ambiguity is removed if we take the square, hence (4.3) makes sense. From subsect. 3.2 we have

$$
(\mathbf{Z} / 2 m \mathbf{Z}) \cong\langle a\rangle \times\langle b\rangle \times \prod_{i=1}^{n}\left\langle a_{i}\right\rangle,
$$


where $a$ has order 2 and $b$ has order $2^{e-1}$. Thus, the solutions to (4.3) have the form

$$
x=a^{\eta_{-1}}\left(b^{2^{c-2}}\right)^{\eta_{0}} \cdot \prod u_{i}^{\eta_{i}} \cdot r,
$$

and there are $2^{n+2}$ solutions. These must be reduced from $(\mathbf{Z} / 2 m \mathbf{Z})$ to $\mathbf{Z} / m \mathbf{Z}$ leaving $2^{n+1}$ solutions. Finally $x$ and $m-x$ are distinct solutions, for $x \in(\mathbf{Z} / m \mathbf{Z})^{*}$, so there are $2^{n}$ solutions to (4.3) for $x \leqslant \phi(m) / 2$. If $n \geqslant 1$ the number of solutions is even and again we can satisfy level matching. To complete the proof of level matching we note that if $m$ is even then $\theta^{m / 2}=-1$ so that (2.6) is trivially satisfied for an even lattice. To summarize, for automorphisms of the form (2.7) (based on primitive $m$ th roots with $\phi(m)=2 d$ ) when (2.2) is satisfied then (2.5) can be satisfied by a proper distribution of the eigenvalues between left and right. In heterotic examples it becomes clear that the two conditions are really distinct: either one may be satisfied without the other.

The next step is the construction of an even self-dual lattice $\Gamma_{d, d}$ with $\mathbf{Z} / m \mathbf{Z}$ symmetry. Our strategy will be simple. Starting with a vector $v \in R^{d, d}$ we consider the rotations of $v$

$$
v, \theta v, \theta^{2} v, \ldots
$$

If $\xi$ is any primitive $m$ th root of unity then it is a root of the cyclotomic polynomial, thus

$$
\theta^{\phi(m)}=-\left[a_{\phi(m)}+\ldots+a_{1} \theta^{\phi(m)-1}\right] .
$$

Hence, the sequence of vectors (4.4) is finite and the span of the vectors $e_{i}=\theta^{i-1} v$, $i=l, \ldots, \phi(m)$ is a lattice with $\mathbf{Z} / m \mathbf{Z}$ symmetry, the automorphism being

$$
\left(\begin{array}{ccccc}
0 & 1 & 0 & 0 & \ldots \\
0 & 0 & 1 & 0 & \ldots \\
0 & 0 & 0 & 1 & \ldots \\
-a_{\phi(m)} & -a_{\phi(m)-1} & . & . & \ldots
\end{array}\right) .
$$

We need the lattice to be $2 d=\phi(m)$ dimensional, even, and self-dual. Thus we compute the quadratic form $e_{i} \cdot e_{j}$ (with the metric $\left(+^{d}-{ }^{d}\right)$ )

$$
Q=\left(\begin{array}{ccccc}
v \cdot v & v \cdot \theta v & v \cdot \theta^{2} v & \ldots & v \cdot \theta^{2 d-1} v \\
& v \cdot v & v \cdot \theta v & \ldots & v \cdot \theta^{2 d-1} v \\
& & v \cdot v \ldots & & \\
& & & & v \cdot v
\end{array}\right)
$$

all the entries of $Q_{i j}$ are related to the first row $a_{i}, i=1, \ldots, 2 d$, by $Q_{i j}=a_{|i-j|+1}$ so we will simply specify $Q_{i j}$ by the vector $a_{i}$. We have no general proof that we can always choose $v$ so that $v \cdot v \in 2 Z$ and $\operatorname{det} Q=1$ (the conditions for even self-duality), but the existence of such choices can be verified explicitly in special cases. We give an example below. A useful observation for manipulating matrices of this type 
is that it can always be block diagonalized to two $d \times d$ blocks using the basis ${ }^{\star}$

$$
\begin{array}{ll}
f_{i}=\left(e_{i}+e_{2 d+1-i}\right) / \sqrt{2}, & i=1, \ldots, d, \\
f_{i}=\left(e_{i}-e_{2 d+1-i}\right) / \sqrt{2}, & i=d+1, \ldots, 2 d .
\end{array}
$$

\section{An example}

We have seen that the condition (2.2) shows that the first allowed twist of the form (2.7) occurs for $m=12$. Since $\phi(12)=4$ we attempt to construct a $\Gamma^{2,2}$ even self-dual lattice using the method described above. The automorphism is taken to be

$$
\theta=\left(\begin{array}{llll}
\xi & & & \\
& \xi^{11} & & \\
& & \xi^{5} & \\
& & & \xi^{7}
\end{array}\right),
$$

where $\xi=\mathrm{e}^{2 \pi i / 12}$, and in a lattice basis is

$$
\left(\begin{array}{rrrr}
0 & 1 & 0 & 0 \\
0 & 0 & 1 & 0 \\
0 & 0 & 0 & 1 \\
-1 & 0 & 1 & 0
\end{array}\right) .
$$

We choose $v$ of the form $v=\left(\begin{array}{llll}v_{1} & 0 & v_{2} & 0\end{array}\right)$ and we may define

$$
\begin{aligned}
a & =v_{1}^{2}-v_{2}^{2}, \\
b & =v \cdot \theta v=v_{1}^{2} \cos (\pi / 6)-v_{2}^{2} \cos (5 \pi / 6) \\
& =(\sqrt{3} / 2)\left(v_{1}^{2}+v_{2}^{2}\right) .
\end{aligned}
$$

Since $v_{1}^{2}, v_{2}^{2}$ are nonnegative numbers we require $4 b^{2} \geqslant 3 a^{2}$. Moreover, one finds $Q=\left(\begin{array}{lll}a & b & a / 2\end{array}\right)$ (using the notation explained in sect. 2) and, block diagonalizing,

$$
\begin{aligned}
\operatorname{det} Q & =\operatorname{det}\left(\begin{array}{cccc}
a & b+a / 2 & & \\
b+a / 2 & a+b & a & b-a / 2 \\
& & b-a / 2 & a-b
\end{array}\right) \\
& =\frac{\left(3 a^{2}-4 b^{2}\right)^{2}}{16} .
\end{aligned}
$$

Choosing $a=b=2$ gives an even self-dual $(2,2)$-lattice with $\mathbf{Z} / 12 \mathbf{Z}$ symmetry.

^ We thank K. Rabe for pointing this out. 
We discuss now the massless scalars in the theory. As a preliminary we demonstrate that when $p_{\mathrm{L}}^{2}$ is not zero then it is irrational. The general momentum vector $\left(p_{\mathrm{L}}, p_{\mathrm{R}}\right)$ can be written $\sum_{i=1}^{4} n_{i} e_{i}$ for $n_{i} \in \mathbf{Z}$. Thus $p_{\mathrm{L}}^{2}=v_{1}^{2} \sum_{k, l=1}^{4} n_{k} n_{l} \cos [(k-$ l) $2 \pi / 12]$. Since $v_{1}^{2}=1+(2 / \sqrt{3})=1+\frac{2}{3}\left(\xi+\left(\xi^{-1}\right), p_{\mathrm{L}}^{2}\right.$ is the value for a vector of integers of a quadratic form over $\mathbf{Q}[\alpha]$, where $\alpha=\xi+\xi^{-1}$. This extension clearly has degree two since $\alpha^{2}=3$ so we can write

$$
\begin{aligned}
p_{\mathrm{L}}^{2} & =\boldsymbol{n} \cdot\left[\left(1+\frac{2}{3} \alpha\right)\left(\begin{array}{llll}
1 & \frac{1}{2} \boldsymbol{\alpha} & \frac{1}{2} & 0
\end{array}\right)\right] \cdot \boldsymbol{n} \\
& =\boldsymbol{n} \cdot\left[\left(\begin{array}{llll}
1 & 1 & \frac{1}{2} & 0
\end{array}\right)+\left(\frac{1}{6} \boldsymbol{\alpha}\right)\left(\begin{array}{llll}
4 & 3 & 2 & 0
\end{array}\right)\right] \cdot \boldsymbol{n}
\end{aligned}
$$

since the quadratic form ( $\left.\begin{array}{llll}4 & 3 & 2 & 0\end{array}\right)$ can be block diagonalized to

$$
\left(\begin{array}{llll}
4 & 5 & & \\
5 & 7 & & \\
& & 4 & 1 \\
& & 1 & 1
\end{array}\right),
$$

which is positive definite we see that $p_{\mathrm{L}}^{2}$ is never rational, if it is non-zero. Since $p_{\mathrm{L}}^{2}-p_{\mathrm{R}}^{2} \in 2 \mathrm{Z}$, the same is true of $p_{\mathrm{R}}^{2}$.

We can finally discuss the spectrum of massless scalars. In the untwisted sector, since we never have $p_{\mathrm{L}}^{2}$ or $p_{\mathrm{R}}^{2}$ equal to two, the only massless scalars have the form

$$
\alpha_{-1}^{i}|0\rangle_{\mathrm{R}} \otimes \alpha_{-1}^{j}|0\rangle_{\mathrm{L}}, \quad i, j=1,2 .
$$

But these are projected out by the twist (2.7). Thus, there are no massless untwisted states. We see here a general feature of quasicrystalline orbifolds. In the case of symmetric orbifolds one can have a twist with the same eigenvalues on the left- and the right-movers. Then oscillator states will survive the projection. Such a twist is impossible in the quasicrystalline case (which is always asymmetric). Some oscillator states will always be projected out. In addition, we see from the above example that the absence of $p^{2}=2$ vectors can eliminate all massless untwisted scalars.

In the twisted sectors we must check if there are scalars constructed by acting on the twisted vacuum with fractionally moded oscillators. In the sector twisted by $\theta$ the left-moving vacuum energy is

$$
E_{\mathrm{L}}=-1+\frac{11}{288}
$$

and the oscillators have moding which are multiples of $1 / 12$. Thus no states have $E_{\mathrm{L}}=0$. The reader can check that in the other sectors there is a similar mismatch between vacuum energy and moding, so that no massless scalars exist. This finally substantiates our first claim, that quasicrystalline compactification can give rise to isolated solutions of the bosonic string. Unfortunately, the mismatch between 
moding and vacuum energy does not seem to be a general feature of the orbifolds we have constructed. In these other cases one must perform scattering amplitude calculations to decide whether twisted scalars are moduli of the theory.

We now discuss the issue of rational approximation. In an orbifold compactification the primary fields are combinations of the oscillators, momentum eigenstates, and twist fields:

$$
\left(\partial^{n} X\right)^{m}, \quad \mathrm{e}^{i p \cdot X}, \quad T_{\eta} .
$$

The rational approximation orbifold must be made from a $(2,2)$ lattice, and as we have seen the left- and right-moving momenta themselves must form lattices. In the first twisted sector of the quasicrystalline orbifold there is a primary field $\phi$ with

$$
(h, \bar{h})=\left(\frac{35}{288}, \frac{35}{288}\right)
$$

and since it is in the first twisted sector and satisfies level matching it is not projected out. This state must be approximated by some primary field $\Phi$ in the rational orbifold. The field $\Phi$ must be either twisted or untwisted. We consider the two cases separately.

If $\Phi$ is untwisted then, since oscillators in the untwisted sector have integral moding, $\Phi$ must be of the form $\mathrm{e}^{i p \cdot X}$ (or some linear combinations of such operators which is group invariant) with

$$
\left(\frac{1}{2} p_{\mathrm{L}}^{2}, \frac{1}{2} p_{\mathrm{R}}^{2}\right) \sim\left(\frac{35}{288}, \frac{35}{288}\right) .
$$

Recall that the definition of approximation includes approximation of operator product expansions. Since

$$
\left[\mathrm{e}^{i p \cdot X}\right]\left[\mathrm{e}^{i p \cdot X}\right] \sim\left[\mathrm{e}^{i 2 p \cdot X}\right]+\ldots,
$$

and $\phi \cdot \phi$ factorizes on the states in the $\theta^{2}$ sector, and since there is a one-one correspondence of primary fields we see that there must be a state of approximate weight

$$
\left(\frac{1}{2}\left(2 p_{\mathrm{L}}\right)^{2}, \frac{1}{2}\left(2 p_{\mathrm{R}}\right)^{2}\right) \sim\left(\frac{140}{288}, \frac{140}{288}\right)
$$

in the quasicrystalline sector $\mathscr{H}_{\theta^{2}}$. In this sector

$$
E_{\mathrm{R}}=-1+\frac{20}{288} .
$$

Since the difference of the right-moving weights is $5 / 12$ and the moding is only $1 / 6$ in $\mathscr{H}_{\theta^{2}}$ we see that there is no corresponding state in the quasicrystalline Hilbert space. Thus $\Phi$ cannot be an untwisted state.

We now consider the possibility that $\Phi$ is a twisted state. First, recall from the introduction that the lattice in a rational orbifold always has a sublattice of the form 
$(p, 0)$. Hence the orbifold group must act crystallographically in $d$ dimensions. Therefore, if $\Phi$ is a twisted state then, given the allowed crystallographic actions in two dimensions, it must be a twist field with $\eta=1 / 2,1 / 3,1 / 4,1 / 6$. With the exception of the order two twist field (with a single -1 eigenvalue), there cannot be an accompanying shift, because the shift can always be taken to be in the invariant sublattice. The corresponding weights are $\frac{1}{2} \eta(1-\eta)$, that is

$$
\begin{array}{ll}
T_{1 / 2}: & h=\frac{36}{288}, \\
T_{1 / 3}: & h=\frac{32}{288}, \\
T_{1 / 4}: & h=\frac{27}{288}, \\
T_{1 / 6}: & h=\frac{20}{288} .
\end{array}
$$

Even taking into account the oscillators none of these fields can approximate the state $\phi$. We now consider the order two twist with a single -1 eigenvalue, which might be combined with a shift. If

$$
\Phi=\mathrm{e}^{i \delta \cdot x} T_{1 / 2}
$$

(where $\delta$ has a component only along the invariant direction of the -1 reflection) then we must have

$$
\frac{1}{2} \delta_{\mathrm{L}}^{2}+\frac{1}{16}-\frac{35}{288} .
$$

If we consider the class $[\Phi]^{4}$ we find there is a field $\mathrm{e}^{4 i \delta \cdot X}$ of weight $16 \frac{1}{2} \delta_{\mathrm{L}}^{2}=\frac{272}{288}$. Since the ground state energy in $\mathscr{H}_{\theta^{4}}$ is

$$
\frac{32}{288} \text {, }
$$

the difference is $5 / 6$, but there are only $1 / 3$ moded oscillators in this sector. This finally substantiates the second claim in the introduction. Unfortunately the argument relied on many details of the model. Intuitively we expect the result since we are twisting by a symmetry which cannot exist for a rational lattice. We should also point out that we have not ruled out the possibility that this theory can be approximated by a rational orbifold based on a quantum symmetry which occurs only at special points in the moduli space of $c=2$ toroidal theories. We cannot analyze this possibility in the absence of a classification of such quantum symmetries.

Before going on to discuss some heterotic examples, let us note that the above example can be used to give a simple type II model. We simply take two copies of the above lattice and mod out by a simultaneous $Z / 12 Z$ rotation on both lattices. In this way we can obtain a four-dimensional compactification of a type II string, and depending on how we lift this action to the fermions (i.e. whether we allow twists by $(-1)^{\mathrm{F}}$ ) we can obtain a theory with 2,1 or no supersymmetries in six dimensions. We do get some massless scalars, and we have not checked whether or not they are massless moduli. 


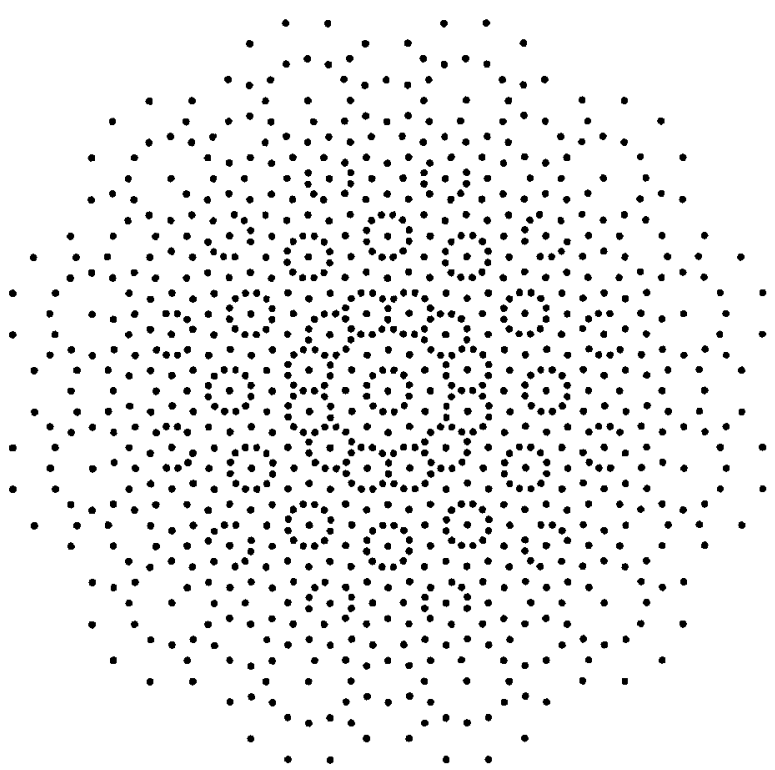

Fig. 1. $p_{\mathbf{L}}$ in the $\mathbf{Z} / 12 \mathrm{Z}$ quasicrystal for states with $p_{\mathbf{L}}^{2}+p_{\mathbf{R}}^{2}<12$.

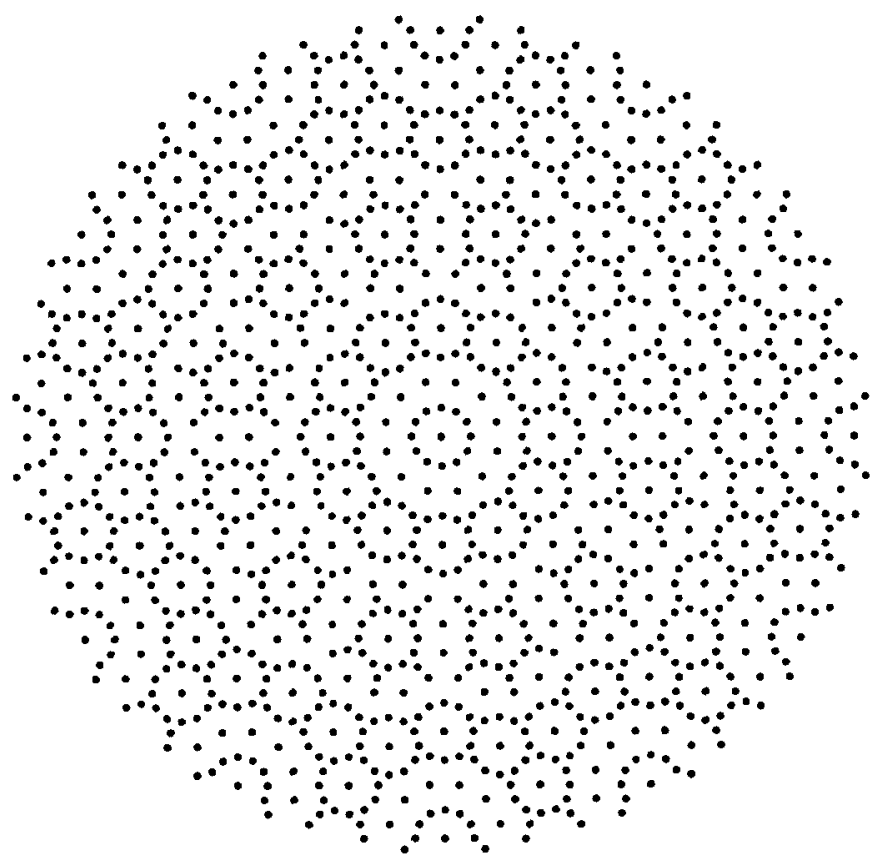

Fig. 2. $p_{\mathrm{L}}$ in the $\mathbf{Z} / 12 \mathbf{Z}$ quasicrystalline orbifold for states with $p_{\mathrm{R}}^{2}<3$. 
For the amusement of the reader we have plotted values of $p_{\mathrm{L}}$ in the $\mathbf{Z} / 12 \mathrm{Z}$ orbifold for $p_{\mathrm{L}}^{2}+p_{\mathrm{R}}^{2}<15$ in fig. 1 and for $p_{\mathrm{R}}^{2}<4$ and $p_{\mathrm{L}}^{2}<32$ in fig. 2 .

\section{Heterotic examples}

It is possible to generalize the bosonic constructions in a variety of ways to give quasicrystalline compactifications of the heterotic string. The first possibility we will consider is to mimic the bosonic construction, but applied to lattices of the type $\Gamma^{16+d, d}$. For simplicity we consider only $d=2,4,6$. For $\Gamma^{18,2}$ we look for solutions to $\phi(m)=20$ and twist by $m$ th roots of unity so that the twist leaves no invariant lattice. There are only five solutions of $\phi(m)=20$, and of these only $m=33,44,66$ can satisfy (2.2). It is an easy exercise ${ }^{\star}$ to check that none of these satisfy level matching. Considering $\Gamma^{22,6}$ there are only two solutions to $\phi(m)=28$, both of which are ruled out by (2.2). Finally, for $\Gamma^{20,4}$ there are 10 solutions to $\phi(m)=24$, namely $m=35,39,45,52,56,70,72,78,84,90$, none of which are ruled out by $(2.2)$. However, level matching allows only $m=52$ (with $\theta_{\mathrm{R}}=\left(\xi \xi^{5} \xi^{51} \xi^{47}\right)$ ). We presume that an even self-dual lattice $\Gamma^{20,4}$ with $\mathbf{Z} / 52 Z$ symmetry exists, but we have not constructed one.

For phenomenological purposes one would like the twist $\theta$ to leave a lattice $I \subset \Gamma^{16+d, d}$ invariant. Points $\left(p_{\mathrm{L}}, p_{\mathrm{R}}\right)$ in $I$ with $\left(p_{\mathrm{L}}^{2}, p_{\mathrm{R}}^{2}\right)=(2,0)$ will then correspond to gauge bosons in the compactified theory. We will discuss two types of compactifications with $I \neq 0$.

In the first we take $\Gamma^{16+d, d}=\Gamma_{d, d} \oplus \Gamma^{16,0}$ with $\Gamma^{16,0}$ equal to $\Gamma^{8} \oplus \Gamma^{8}$ or $\Gamma^{16}$, the two even self-dual euclidean lattices of rank 16. For $\Gamma_{d, d}$ we take one of the lattices with quasicrystalline symmetry constructed in sect. 2 . Twisting by this symmetry will remove the massless scalars which correspond to the usual Narain moduli. In general, however, there will be massless scalars in twisted sectors and a calculation is required to determine whether these correspond to moduli.

The conditions for level matching are different than in the bosonic case since we must now twist the right-moving fermions as well. To maintain modular invariance we will have to accompany the $\mathbf{Z} / m \mathbf{Z}$ twist by a shift $\delta$ with $m \delta \in \Gamma^{16,0}$. If $\theta_{\mathrm{L}}=$ $\left(\xi^{l_{1}} \xi^{m-l_{1}} \ldots \xi^{l_{d / 2}} \xi^{m-l_{d / 2}}\right) \theta_{\mathrm{R}}=\left(\xi^{r_{1}} \xi^{m-r_{1}} \ldots \xi^{r_{d / 2}} \xi^{m-r_{d / 2}}\right)$ then level matching requires

$$
\begin{aligned}
\sum_{a=1}^{d / 2} l_{a}^{2}+(m \delta)^{2} & =0 \begin{cases}\bmod m & m \text { odd } \\
\bmod 2 m & m \text { even }\end{cases} \\
\sum r_{a} & =0 \bmod 2 .
\end{aligned}
$$

Another way to apply these ideas to the heterotic string is to start with a lattice of the form $\Gamma^{d, r} \oplus \Gamma^{0,16+d-r}$ where $\Gamma^{d, r}$ is an even lattice constructed using the

^ Using a computer! 
TABLE 1

\begin{tabular}{lll}
\hline Symmetry & $\Gamma^{d, r}$ & $I$ \\
\hline $\mathbf{Z} / 7 \mathbf{Z}$ & $\Gamma^{2,4}$ & $\mathrm{E}_{8} \otimes \mathrm{A}_{6}$ \\
$\mathbf{Z} / 9 \mathbf{Z}$ & $\Gamma^{2,4}$ & $\mathrm{E}_{8} \otimes \mathrm{E}_{6}$ \\
$\mathbf{Z} / 13 \mathbf{Z}$ & $\Gamma^{4,8}$ & $\mathrm{~A}_{12}$ \\
$\mathbf{Z} / 25 \mathbf{Z}$ & $\Gamma^{4,16}$ & $\mathrm{~A}_{4}$ \\
$\mathbf{Z} / 11 \mathbf{Z}$ & $\Gamma^{6,4}$ & $\mathrm{E}_{8} \otimes \mathrm{A}_{10}$ \\
$\mathbf{Z} / 27 \mathbf{Z}$ & $\Gamma^{6,12}$ & $\mathrm{E}_{8} \otimes \mathrm{A}_{2}$ \\
$\mathbf{Z} / 17 \mathbf{Z}$ & $\Gamma^{6,8}$ & $\mathrm{~A}_{12}$ \\
\hline
\end{tabular}

rotation method with $\mathbf{Z} / m \mathbf{Z}$ symmetry where $\phi(m)=d+r . \Gamma^{0,16+d-r} \equiv I$ will be an even lattice which is left invariant by the twist. In general $\Gamma^{d, r} \oplus \Gamma^{0,16+d-r}$ will not be self-dual, but it is often possible to construct an even self-dual lattice from it by a "gluing" procedure. That is, we attempt to add even vectors of the form $\left(e_{i}^{*}, f_{i}^{*}\right)$ where $e^{*} \in \Gamma^{d, r^{*}}, f^{*} \in \Gamma^{0,16+d-r^{*}}$ so that $\Gamma^{d, r} \oplus \Gamma^{0,16+d-r}+\sum_{i}\left(e_{i}^{*}, f_{i}^{*}\right)$ is even and self-dual. This will be illustrated later in an example.

Let us now discuss the conditions on $d, r$ and $m$. If $d-r \neq 0(8)$ then $I$ is not self-dual and hence $\left|I^{*} / I\right| \neq 1$. So modular invariance requires that $\operatorname{det}(1-\theta) \neq 1$ where $\theta$ is the generator of the $\mathbf{Z} / m \mathbf{Z}$ symmetry we wish to mod out by. Thus, for $d-r \neq 0(8),(2.2)$ requires $m=p^{r}$ for $p$ prime and we have $\operatorname{det}(1-\theta)=p$ so that $\left|I^{*} / I\right|=p$. We also need to check (2.2) for powers of $\theta$. It is sufficient to check $\theta^{p^{s}}$ for $s<r$. Now $\operatorname{det}\left(1-\theta^{p^{s}}\right)=p^{p^{s}}$ so (2.2) requires that $p^{p^{s}-1}$ be a square which is true as long as $p$ is an odd prime.

To construct explicit examples it is useful to take $I$ to be a tensor product of A, $\mathrm{D}$, or E root lattices. Table 1 lists some possibilities for $\Gamma^{d, r}$ and $I$.

To illustrate the procedure let us explicitly construct the first example in table 1 . Let $\xi=\mathrm{e}^{2 \pi i / 7}$ and consider the twist

$$
\begin{aligned}
& \theta_{\mathrm{L}}=\operatorname{diag}\left(\begin{array}{llll}
\xi^{2} & \xi^{5} & \xi^{3} & \xi^{4}
\end{array}\right) \\
& \theta_{\mathrm{R}}=\operatorname{diag}\left(\begin{array}{ll}
\xi & \xi^{6}
\end{array}\right)
\end{aligned}
$$

in $\mathbf{R}^{2,4}$. To construct a lattice $\Gamma^{2,4}$ with $\mathbf{Z} / 7 \mathbf{Z}$ symmetry we proceed as before. Choosing an appropriate vector $v$ in $\mathbf{R}^{2,4}$ we can form a lattice with basis

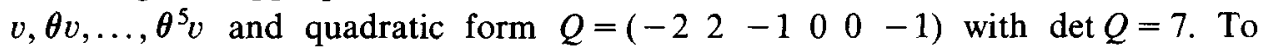
make $\Gamma^{2,4} \oplus I$ self-dual (with $I=\Lambda_{\text {root }}\left(\mathrm{E}_{8} \otimes \mathrm{A}_{6}\right)$ ) we choose an element

$$
e_{1}^{*}=\frac{1}{7}\left(-4 e_{1}-e_{2}+2 e_{3}-2 e_{4}-6 e_{5}-3 e_{6}\right)
$$

in $\left(\Gamma^{2,4}\right)^{*}$ with $\left(e_{1}^{*}\right)^{2}=-4 / 7$, together with a weight vector $f^{*}$ of $\mathrm{SU}(7)$ with $\left(f^{*}\right)^{2}=-10 / 7$. If we add the shifted lattice $\Gamma^{2,4} \oplus \Lambda_{r}(\mathrm{SU}(7))+e_{1}^{*} \oplus f^{*}$ to $\Gamma^{2,4} \oplus$ 
$\Lambda_{r}(\mathrm{SU}(7))$ we obtain an even self-dual lattice of signature $(2,10)$. Self-duality is proved by considering the inner product of a dual vector $\left(v_{1}, v_{2}\right)$ with $(p, 0)(0, p)$ and $\left(e_{1}^{*}, f^{*}\right)$.

In order to satisfy modular invariance we accompany the twist with a shift in the $\mathrm{E}_{8}$ lattice by a vector $\delta$ satisfying $(7 \delta)^{2}=6$. (For example, choose $7 \delta=$ $\left(\begin{array}{llll}-2 & 1 & 1 & 0^{5}\end{array}\right)$.) One can show, as before, that $p_{\mathrm{R}}^{2}$ is never rational, if non-zero, a fact which eliminates many possible massless scalars. Still, some scalars remain, so our goal has only been partially achieved. Again, one might expect that this orbifold is not approximable by a rational orbifold, although we have not carefully checked this. We will not discuss this model further.

\section{Conclusion}

We have seen that quasicrystalline compactification of the bosonic string provides a neat set of examples of isolated non-rationally approximable orbifolds. In addition the quasicrystalline compactifications can be discussed for type II superstrings, as well as heterotic strings. Some concrete examples of these were discussed in this paper. We have not determined whether any of the supersymmetric compactifications are isolated.

We should mention that the issue of rational approximation is not completely settled by our arguments. We have shown that bosonic quasicrystals cannot be approximated by rational orbifolds but it is conceivable that they can be approximated by some other class of RCFTs.

It is also important to note that even though our constructions are the only known examples of isolated irrational conformal theories, there are many known isolated rational conformal theories. The discrete series with central extension $c<1$ provides such an infinite set of examples (provided one can make sense of them on higher genus Riemann surfaces). Motivated by the Moonshine module [11], an example of an isolated rational asymmetric orbifold can be made by combining the right-movers of the heterotic string (or the bosonic string for that matter) with the left-movers on an orbifold formed from the Leech torus and the automorphism $x \rightarrow-x$. The identity sector has no massless moduli because all the massless oscillator states are projected out, and there are no massless momentum states because the smallest non-vanishing vector of the Leech lattice has (length) ${ }^{2}=4$. In the twisted sector, the zero-point energy is too high to allow a massless state. This provides an example of a rational orbifold with no massless state ${ }^{\star}$. Other examples of theories with no massless moduli, based on rational orbifolds, have been

* Not even a dilaton! It may be interesting to see what, even in principle, fixes the coupling constant for the string theory in this example, because it is commonly believed that the expectation value for the dilaton field gives the value of the coupling constant, which cannot be the case in this example. 
constructed by Dixon [12], and Vafa [13]. Some examples of isolated rational conformal theories at $c=1$ have been discussed by Ginsparg [14].

These orbifolds also raise some interesting questions in the fermionic formulation of orbifolds. It is known that one can often rewrite these theories either in terms of bosonic or fermionic degrees of freedom. However, if one wishes to work in terms of free fermions then the fermions obey twisted boundary conditions of finite order only if the corresponding background data in the bosonic theory are rational. For irrational values it is not really clear whether the fermionic formulation makes sense or is useful. If one then further twists the fields as we have done here it becomes even less clear how one would treat these theories in a fermionic formulation.

We would like to thank D. Friedan, K. Rabe and S. Shenker for discussions. C. Vafa would like, in addition, to thank H. Aoyama for discussions about quasicrystals. J.H. and G.M. would like to thank the Aspen Center for physics for its hospitality while this work was being completed. J.H. was supported in part by NSF grant PHY80-19754, the A.P. Sloan Foundation, and a NSF PYI award PHY8658033. G.M. was supported in part by DOE grant no. DEACO276ER02220. C.V. was supported in part by NSF grant PHY82-15249 and the Harvard Society of Fellows.

\section{Appendix}

\section{ALTERNATIVE DEFINITIONS OF RATIONALITY}

In this appendix we give some alternative definitions of rational conformal theories and discuss their relations.

The most obvious choice is

Definition 1. The value of $c$ and all conformal weights $\left\{h_{i}\right\}$ for primary fields are rational numbers.

It is conceivable that some restriction on the operator product coefficients should be imposed. Less obvious, but perhaps more useful, is the definition used in the paper.

Definition 2. The partition function on a flat torus with modular parameter $\tau$ is modular invariant and has the form

$$
Z(\tau, \bar{\tau})=\sum_{i=1}^{N} f_{i}(\tau) \bar{g}_{i}(\tau),
$$

where $f_{i}, g_{i}$ are holomorphic and $N<\infty$. This definition should probably be strengthened to include a restriction on the form of higher loop partition functions, but we will not discuss these issues. Another way one can strengthen definition 1 (D1) is by requiring

Definition 3. The partition function on the flat torus has the form (A.1) where $f_{i}, g_{i}$ are modular forms for some subgroup $\Gamma^{\prime} \subseteq \operatorname{PSL}(2, \mathbf{Z}) \equiv \Gamma$. 
Definition 3 (D3) is equivalent to total holomorphic factorization of $Z(\tau, \bar{\tau})$ on some covering $\mathscr{H} / \Gamma^{\prime}$ of moduli space $\mathscr{H} / \Gamma$. The generic number of sheets of this covering is just the index $\left[\Gamma: \Gamma^{\prime}\right]$.

For example, it can be shown that orbifolds based on rational tori are rational with this definition. One need only show that the theta functions of (1.2) are modular forms for some congruence subgroup of the modular group. This follows from a theorem of Schoeneberg [15].

We now wish to comment on the possible equivalence of these definitions. Again, the absence of a deep understanding of the space of conformal theories hinders the analysis of this question.

Obviously D3 implies (D2). If $\left[\Gamma: \Gamma^{\prime}\right]<\infty$ then it is clear that D3 implies D1 since the powers of $q$ in a modular form are rational.

Similarly, if there are a finite number of primary fields (which can only happen if $c<1$ [16]), or more generally if all the values of the $h_{i}$ have a largest denominator, then D1 implies D2. If there are an infinite number of values of $h_{i}$ with growing denominators then this implication might fail.

We now consider the converse statement, namely, does D2 imply D1? If $N=1$ we have total holomorphic factorization. By modular invariance, $f, g$ must form a one-dimensional representation $\rho$ of the modular group. This group has two generators $S$ and $T$ satisfying the relations $S^{2}=1,(S T)^{3}=1$ ( $S$ is represented by $\tau \rightarrow-1 / \tau$ and $T$ by $\tau \rightarrow \tau+1)$. Thus $\rho(S)$ is a square root, and $\rho(S T)$ is a cube root of one. Hence $\rho(T)$ is a sixth root of one and hence $h$ is rational. The above argument does not generalize. Indeed, consider a two-dimensional unitary representation of $\Gamma$ obtained by choosing "rotation matrices" $U_{1}, U_{2}$ of orders 2 and 3 respectively whose "axis of rotation" form an angle $\theta$ with $\cos (\theta)$ irrational. If we set $\rho(S)=U_{1}$ and $\rho(S T)=U_{2}$ then the eigenvalues of $\rho(T)=U_{1} U_{2}$ will be irrational. We may, in fact, form a pair of holomorphic functions on $\mathscr{H}$ transforming according to $\rho$ using a Poincaré series*

$$
\left(\begin{array}{l}
F_{1} \\
F_{2}
\end{array}\right)(\tau)=\sum_{\gamma \in \Gamma} \rho\left(\gamma^{-1}\right)\left(\begin{array}{l}
f_{1}(\gamma \tau) \\
f_{2}(\gamma \tau)
\end{array}\right) \cdot(c \tau+d)^{-k} .
$$

For reasonable functions $f_{i}$ and sufficiently large $k$ the series ought to converge ${ }^{\star \star}$, defining "modular forms" $F_{1}, F_{2}$ of weight $k$ transforming in the representation $\rho$. Dividing by $\eta^{k}$ provides a representation of $\Gamma$ which would violate the implication $\mathrm{D} 2 \rightarrow \mathrm{D} 1$. It is unclear whether such functions can arise in a conformal field theory. In particular it is not clear whether the coefficients in front of $q^{a} \bar{q}^{b}$ are integers, as is required for a partition function coming from a conformal theory ${ }^{\dagger}$.

\footnotetext{
\#e would like to thank Martinec for suggesting this construction.

$\star$ * We have not proven that it does.

${ }^{\dagger}$ Note added in proof: The implication $\mathrm{D} 2 \Rightarrow \mathrm{D} 1$ has been demonstrated in ref. [17]. An alternative argument for rationality of $c, h$ in RCFT can be found in ref. [18].
} 


\section{References}

[1] D. Friedan and S. Shenker, unpublished

[2] G. Moore, HUTP-A013, Nucl. Phys. B293 (1987) 139

[3] P. Ginsparg and C. Vafa, Nucl. Phys. B289 (1987) 414

[4] K.S. Narain, M.H. Sarmadi, and C. Vafa, Nucl. Phys. B288 (1987) 551

[5] K.S. Narain, Phys. Lett. B169 (1986) 41

[6] K.S. Narain, M.H. Sarmadi, and E. Witten, Nucl. Phys. B279 (1986) 369

[7] D. Freed and C. Vafa, Comm. Math. Phys. 110 (1987) 349

[8] K.S. Narain et al., to appear

[9] I.N. Stewart and D.O. Tall, Algebraic number theory, (Chapman and Hall, 1987)

[10] K. Ireland and M. Rosen, A classical introduction to modern number theory, (Springer, Berlin, 1982)

[11] I. Frenkel, J. Lepowsky, and A. Meurman, in Vertex operators in mathematics and physics, eds., J. Lepowsky, S. Mandelstam, and I. Singer, MSRI publications No. 3

[12] L. Dixon, unpublished

[13] C. Vafa, unpublished

[14] P. Ginsparg, Nucl. Phys. B295[FS21] (1988) 153

[15] B. Schoeneberg, Elliptic modular functions (Springer, Berlin, 1974)

[16] J. Cardy, Nucl. Phys. B270 (1986) 186

[17] G. Anderson and G. Moore, Rationality in conformal field theory, IASSNS-HEP-87/69, Commun. Math. Phys., to appear

[18] C. Vafa, Toward classification of conformal theories, HUTP-88/A011 